\title{
State- and conformer-selected beams of aligned and oriented molecules for ultrafast diffraction studies $\dagger$
}

\author{
Frank Filsinger, ${ }^{a}$ Gerard Meijer $^{a}{ }^{\text {Henrik Stapelfeldt, }{ }^{b} \text { Henry N. Chapman }}{ }^{c d}$ and \\ Jochen Küpper $\neq * a$
}

Received 24th August 2010, Accepted 1st November 2010

DOI: $10.1039 / \mathrm{c0cp01585g}$

The manipulation of the motion of neutral molecules with electric or magnetic fields has seen tremendous progress over the last decade. Recently, these techniques have been extended to the manipulation of large and complex molecules. In this article we introduce experimental approaches to the manipulation of large molecules, i.e., the deflection, focusing and deceleration using electric fields. We detail how these methods can be exploited to spatially separate quantum states and how to select individual conformers of complex molecules. We briefly describe mixed-field orientation experiments made possible by the quantum-state selection. Moreover, we provide an outlook on ultrafast diffraction experiments using these highly controlled samples.

${ }^{a}$ Fritz-Haber-Institut der Max-Planck-Gesellschaft, Faradayweg 4-6, 14195 Berlin,Germany.E-mail: jochen@fhi-berlin.mpg.de

${ }^{b}$ Aarhus University, Department of Chemistry and Interdisciplinary Nanoscience Center (iNANO), 8000 Aarhus C, Denmark

${ }^{c}$ Center for Free Electron Laser Science, DESY, Notkestrasse 85, 22607 Hamburg, Germany

${ }^{d}$ University of Hamburg, Luruper Chaussee 149, 22761 Hamburg, Germany

$\dagger$ Nernst-Haber-Bodenstein award 2009 of the German Bunsen Society for Physical Chemistry for Jochen Küpper.

$\ddagger$ Present address: Center for Free Electron Laser Science, DESY, Notkestrasse 85, 22607 Hamburg, Germany and University of Hamburg, Luruper Chaussee 149, 22761 Hamburg, Germany. jochen.kuepper@cfel.de

\section{Introduction}

A Manipulation of molecular beams with electric and magnetic fields

1 Deflection of polar molecules. By expanding atoms or molecules from a reservoir at high pressure into vacuum a so called atomic or molecular beam is created. In such beams the molecules' intrinsic properties can be investigated under collision-free conditions, independent from interactions with other species. A century ago, when such beams were initially investigated, ${ }^{1}$ laser-based quantum-state-selective detection

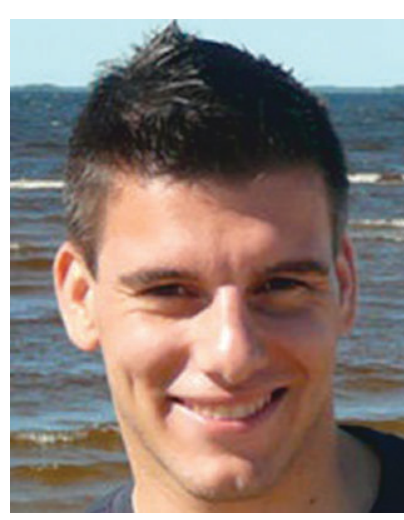

Frank Filsinger
Frank Filsinger, 31, studied physics at the University of Heidelberg, Germany, and did his Master studies ("Diplomarbeit") with Prof. Dr U. Platt in the Department of Environmental Physics. In 2005 he joined the group of Jochen Küpper and Gerard Meijer at the Fritz Haber Institute in Berlin as a PhD student working on the manipulation of large neutral molecules with electric fields. He received his Doctoral degree in February 2010 from Radboud University Nijmegen, NL, and currently works as a postdoctoral researcher at the Fritz Haber Institute investigating $X$-ray diffraction of gas-phase molecules with XFELs.

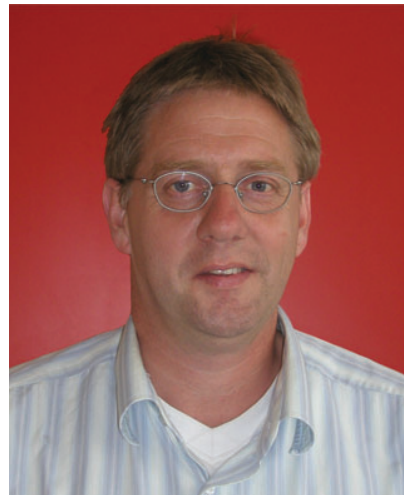

Gerard Meijer
Gerard Meijer, 48, is one of the pioneers of Stark deceleration. In 1995 he became professor in Nijmegen, NL and 2000 director at the FOM Institute for Plasma Physics, Nieuwegein, NL. There he initiated a research program on cold molecules and investigated molecules in the gas-phase using the free electron laser FELIX. Since 2003 he is director of the Department of Molecular Physics at the Fritz Haber Institute of the MPG in Berlin, Germany. 
techniques were still lacking. In 1921 Stern proposed that the trajectories of silver atoms on their way to the detector could be characteristically altered, depending on their quantum state, when the atomic beam was exposed to an inhomogeneous magnetic field. ${ }^{2}$ In a ground-breaking experiment, Gerlach and Stern demonstrated in $1922^{3}$ that indeed quantum-state selectivity could be achieved in the detection process by sorting different quantum states via space quantization, a concept that has been extensively used ever since. The possibility to deflect polar molecules in a molecular beam with electric fields was conceived at the same time. It was first theoretically described by Kallmann and Reiche in $1921^{4,131}$ and later experimentally demonstrated by Wrede - a graduate student of Stern - in $1927 . .^{5}$

As early as 1926, Stern suggested that the technique could be used for the quantum-state separation of small diatomic molecules at low temperatures. ${ }^{6}$ Over the years, various experimental geometries were designed to create strong field gradients on the beam axis in order to efficiently deflect

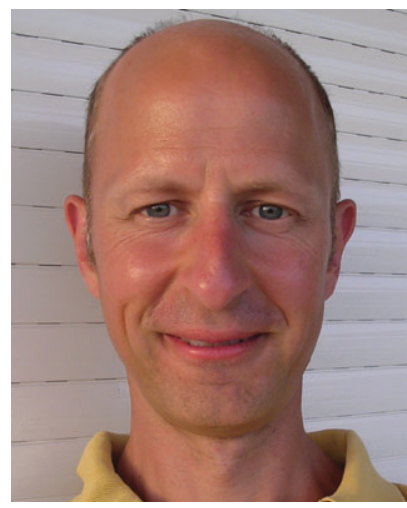

Henrik Stapelfeldt
Henrik Stapelfeldt, 45, is professor at the Chemistry Department, Aarhus University in Denmark. He has pioneered the use of laser pulses, and recently their combination with static electric fields, to control the spatial orientation of molecules. The aligned and oriented molecules are used to study ultrafast chemical reaction dynamics observed with femtosecond time resolved photoelectron spectroscopy or with timed Coulomb explosion imaging. A particular emphasis is on following conformational changes in chiral systems. particles. In 1938/1939 Rabi introduced the molecular beam magnetic resonance method, by using two deflection elements of oppositely directed gradients in succession, to study the quantum structure of atoms and molecules. ${ }^{7,8}$ In his setup, the deflection of particles caused by the first magnet was compensated by a second magnet such that the particles reached the detector on a sigmoidal path. If in between the two magnets a transition to a different quantum state was induced, this compensation was incomplete and a reduction of the detected signal could be observed. For more details on these historic experiments we refer to Ramsey's classical textbook on "Molecular Beams". 9 Since these early days of molecular beam deflection experiments, the deflection technique has been widely used as a tool to determine dipole moments and polarizabilities of molecular systems ranging from diatomics ${ }^{5}$ to clusters ${ }^{10,11}$ to large biomolecules. ${ }^{12}$

2 Focusing and deceleration of molecules in low-fieldseeking quantum states. Whereas deflection experiments allow for the spatial dispersion of quantum states, they do not provide any focusing of the molecular beam. For small molecules in eigenstates whose energy increases with increasing field strength, so-called low-field-seeking (lfs) states, focusing was achieved using multipole focusers. Both magnetostatic ${ }^{13,14}$ and electrostatic ${ }^{15}$ devices were developed in the early 1950s by Paul's group in Bonn. Independently, an electrostatic quadrupole focuser, i.e., a symmetric arrangement of four cylindrical electrodes around the beam axis that are alternately at positive and negative voltage, was built in $1954 / 55$ by Gordon, Zeiger and Townes in New York to create the population inversion of ammonia molecules for the first demonstration of the MASER. ${ }^{16,17}$ Using several multipole focusers in succession and interaction regions with electromagnetic radiation in between them, many setups were developed to unravel the quantum structure of atoms and molecules - very similar to Rabi's molecular beam magnetic resonance method. About ten years after the invention of the multipole focusing technique, molecular samples in a single

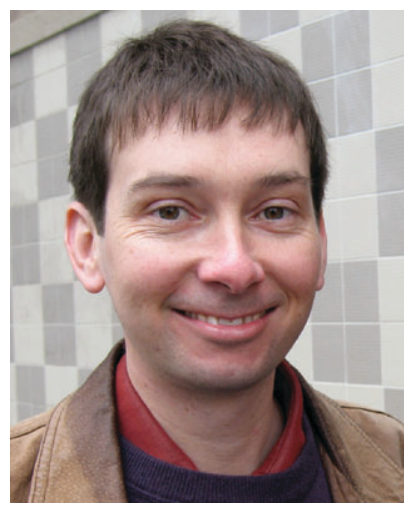

Henry N. Chapman
Henry Chapman, 43, is pursuing molecular and biological coherent imaging using X-ray free-electron lasers, and led efforts to carry out the first imaging experiments at the FLASH FEL in Hamburg and the Linac Coherent Light Source in Stanford. He is investigating the spatial and temporal limits to which objects such as molecules, complexes, and viruses, can be imaged with the "diffraction before destruction" technique, which will open up structure determination to objects that can't necessarily be crystallized. Henry is one of the founding directors of the Center for FreeElectron Laser Science (CFEL) in Hamburg, professor at the University of Hamburg, and leads the CFEL Coherent Imaging Division at DESY.

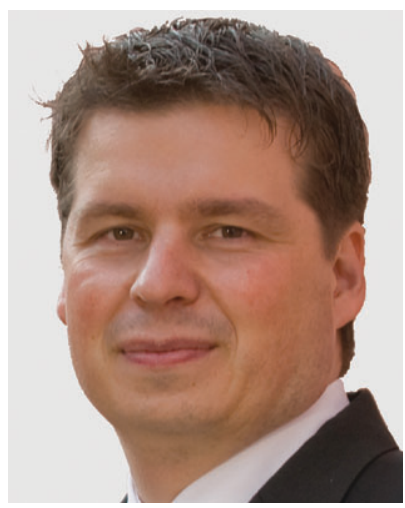

Jochen Küpper
Jochen Küpper, 39, is developing and applying methods to manipulate the motion and to study the dynamics of large molecules. He has performed high resolution laser spectroscopy of molecular aggregates and investigated transient species in superfluid helium droplets. In 2003 he became a group leader at the Fritz Haber Institute in Berlin. Recently, he moved to Hamburg, where he leads the Controlled Molecule Imaging group at the Center of Free-Electron Laser Science, DESY and the University of Hamburg. He continues experiments on the control of large molecules. These controlled samples are then exploited in the development of structural imaging experiments, such as photoelectron angular distribution and electron or X-ray imaging. 
rotational state were used for state-specific inelastic scattering experiments by the Bonn group ${ }^{18}$ and, shortly thereafter, for reactive scattering studies. ${ }^{19,20}$ In the following decades, multipole focusers were extensively used to study steric effects in gasphase reactive scattering experiments. ${ }^{21,22}$ The preparation of oriented samples of state-selected molecules using electrostatic focusers was also essential for the investigation of steric effects in gas-surface scattering ${ }^{23}$ and photodissociation ${ }^{24}$ experiments. Variants of multipole focusing setups were implemented in many laboratories all over the world and yielded important information on stable molecules, radicals, and molecular complexes.

Finally, in 1999 the so-called Stark decelerator was realized, ${ }^{25}$ allowing the same control over the forward velocities of molecules in lfs states. This technique was used to confine small molecules in storage rings ${ }^{26}$ and $\operatorname{static}^{27}$ and dynamic traps. ${ }^{28}$ Recently, the "decelerator on a chip" - a miniaturized version of the Stark decelerator-has been implemented. ${ }^{29}$ Detailed accounts of the field of Stark deceleration have been given elsewhere. ${ }^{30-32}$

3 Focusing and deceleration of molecules in high-fieldseeking quantum states. Large or heavy molecules have small rotational constants and, as a consequence, a high density of rotational states. Coupling between closely spaced states of the same symmetry turns lfs states into hfs states already at relatively weak electric field strengths (compared to the field strengths that are required for efficient focusing). In order to focus molecules in these states, a maximum of the electric field in free space would have to be created. Since Maxwell's equations do not allow for the creation of a 3D maximum with static fields alone, ${ }^{33,34}$ static multipole fields cannot be applied to focus molecules in hfs states. The situation is analogous to charged particle physics: charged particles also cannot be confined with static potentials alone. This focusing problem for ions was solved when Courant, Livingstone, and Snyder introduced the principle of "alternating gradient (AG) focusing" in the $1950 \mathrm{~s} .{ }^{35,36}$ The basic idea is to create an array of electrostatic lenses that focus the particles along one transverse coordinate while defocusing them along the perpendicular transverse axis. Alternating the orientation of these fields at the appropriate frequency results in a net focusing force along both transverse coordinates. This principle is exploited to confine ions, for instance, in quadrupole mass filters, ${ }^{37,38}$ in Paul traps, ${ }^{37,39}$ and in virtually all particle accelerators. The application of $\mathrm{AG}$ focusing to neutral polar molecules was first proposed by Auerbach, Bromberg, and Wharton ${ }^{40}$ and experimentally demonstrated by Kakati and Lainé for ammonia molecules in hfs states. ${ }^{41-43}$ Later, the diatomic $\mathrm{KF}^{44,45}$ and $\mathrm{ICl}^{46}$ molecules were also focused. More recently, slow ammonia molecules were guided from an effusive source using a bent AG focuser, ${ }^{47}$ even though molecules in lfs and hfs states could not be distinguished because the detection process was not state selective. Furthermore, CaF molecules have been guided using a 1 m-long straight $\mathrm{AG}$ focuser. ${ }^{48}$ Besides the $\mathrm{AG}$ focusing technique, various alternative approaches were implemented to focus molecules in hfs states, such as exploiting the fringe fields of ring-like electrode structures, ${ }^{49}$ the fields created by crossed wires, ${ }^{50}$ or the fields created by coaxial electrodes. ${ }^{51-54}$
Most of these methods, however, were only used for proof-ofprinciple experiments and did not find further applications.

The first attempt to manipulate the forward velocity of molecules in hfs states was reported in the 1960s, when the group of Wharton at the University of Chicago set up an 11 m-long machine to accelerate LiF molecules. ${ }^{55,56}$ While these early experiments were unsuccessful and stopped after the PhD student had finished his thesis, a decelerator design that exploits the AG principle for transverse confinement of the molecules was successfully implemented in $2002,{ }^{57}$ inspired by the successful deceleration of small molecules with the Stark decelerator. So-called AG decelerators were used to decelerate $\mathrm{CO},{ }^{57,58} \mathrm{YbF},{ }^{59}$ and benzonitrile ${ }^{60}$ molecules in hfs quantum states and $\mathrm{OH}$ radicals in both hfs and lfs states. ${ }^{61,62}$ In these first experiments on high-field-seeking molecules, up to $30 \%$ of the kinetic energy was removed, but so far it has not been possible to decelerate molecules to velocities that are small enough for trapping in stationary traps.

AC trapping of para- $\mathrm{ND}_{3}$ in the hfs component of its ground rotational state $\left(J_{\mathrm{K}}=1_{1}\right)$ was achieved by decelerating the molecule in a lfs state with a conventional Stark decelerator and subsequently transferring the population to the hfs state using microwave radiation. ${ }^{28}$

Moreover, high-frequency AC fields have also been used for the deflection, focusing, and deceleration of neutral molecules, and these methods are generally applicable to molecules in all, $\mathrm{dc}$ lfs and hfs, states. Strong laser fields have been used to deflect and focus ${ }^{63,64}$ and to decelerate ${ }^{65}$ a fraction of the molecules in a beam. Alternatively, the focusing of molecules with microwave fields has been demonstrated recently. ${ }^{66}$

\section{B Large neutral molecules in the gas phase}

During the last decades, the properties of biomolecules in the gas phase have been studied in ever greater detail. ${ }^{67-69}$ Although the study of biomolecules outside of their natural environment was met with skepticism in the beginning, spectroscopic studies on isolated species in a molecular beam have proven to be very powerful for understanding the molecules' intrinsic properties and for benchmarking theoretical calculations. Moreover, the molecule's native environment can be partly mimicked by adding solvent molecules one by one. ${ }^{69-72}$

Even in the cold environment of a molecular beam, biomolecules exist in various conformational structures. ${ }^{73,74}$ In many cases, the individual conformers are identified via their different electronic spectra. ${ }^{74,75}$ Structural information on the individual conformers can be deduced from, for instance, multipleresonance techniques, which yield conformer-specific infrared spectra. $^{76,77}$ Moreover, one can exploit the different angles between vibrational transition moments and the permanent dipole moments of oriented molecules. ${ }^{78}$ Quadrupole coupling constants, determined by means of Fourier-transform microwave spectroscopy, ${ }^{79}$ or permanent dipole moments, deduced from the rotationally resolved spectra ${ }^{80,81}$ are also conformer specific.

The preparation of conformer-selected samples of biomolecules could enable a new class of experiments to be performed on these systems. For charged species, the separation of structurally different molecules has been demonstrated using ion mobility in drift tubes. ${ }^{82,83}$ For neutral molecules it has been 


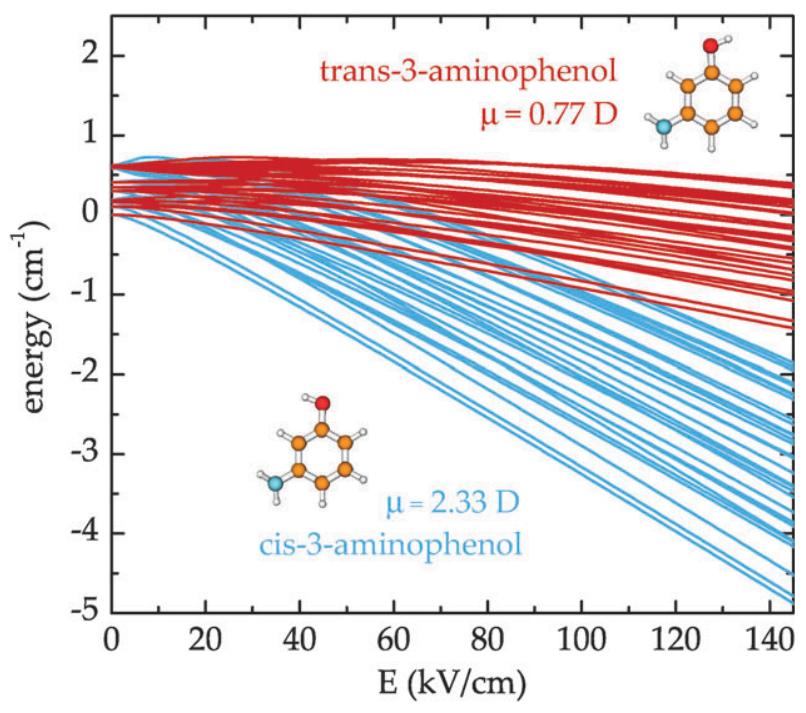

Fig. 1 Molecular structures, dipole moments, and energies of the lowest rotational states of cis- and trans-3-aminophenol as a function of the electric field strength (reproduced from ref. 86).

demonstrated that the abundance of the conformers in the beam can be partly influenced by selective over-the-barrier excitation in the early stage of the expansion ${ }^{84}$ or by changing the carrier gas. ${ }^{85}$ Both methods for neutrals, however, are neither generally applicable nor able to specifically select conformers.

Spatial separation of conformers can be achieved by exploiting their specific interaction with electric fields. All conformers of a molecule have the same mass and the same connectivities between the atoms (primary structure), but often differ by their dipole moments, which are largely determined by the orientations of the functional groups in the molecular frame, i.e., by the folding pattern (secondary structure). These different dipole moments lead to different Stark shifts of the rotational energy levels in an electric field, as shown in Fig. 1 for the prototypical large molecule 3-aminophenol (3AP). The force that a molecule experiences in an electric field is determined by its effective dipole moment $\mu_{\mathrm{eff}}$, which is given by the negative slope of the Stark curve. From Fig. 1 it is obvious that the two conformers of 3-aminophenol will experience different forces in an electric field, which can be exploited to spatially separate them (vide infra).

Such conformer-selected samples are expected to benefit a variety of future applications such as tomographic imaging experiments ${ }^{87}$ or ultrafast dynamics studies on the groundstate potential energy surface. For ultrafast electron and X-ray diffraction experiments ${ }^{88-90}$ aiming at the "molecular movie", i.e., measuring chemical processes with spatial and temporal atomic resolution (a few picometers and femtoseconds, respectively), the preparation of conformer-selected samples might be crucial, as we will see in the remainder of this article.

\section{X-Ray diffractive imaging of molecular ensembles}

Here we discuss the possibility of ultrafast diffraction experiments of controlled samples in a molecular beam. Clearly, $\mathrm{X}$-ray crystallography is at the very heart of structural biology. However, many biological molecules do not crystallize and many cannot easily be purified. The new X-ray Free Electron
Laser (XFEL) light sources ${ }^{91,92}$ promise the possibility to obtain single-molecule diffraction images of large molecules in the gas phase. This could, for example, help biology to obtain structural information on the large number of un-crystallizable proteins and other difficult systems. ${ }^{93}$

It is generally understood that "Nothing tends so much to the advancement of knowledge as the application of a new instrument". 94 The ability to determine the structure of individual biological molecules - using XFEL radiationwithout the need for purification and crystallization would constitute a fundamental breakthrough for structural biology, confirming the above quotation of Sir Humphry Davy. However, the proposed experiments for large molecules rely on the recording of a detectable diffraction pattern from a single molecule in order to be able to classify and average images from multiple shots. ${ }^{95}$ It is not a priori clear whether it is possible to obtain such a single-molecule diffraction image at all, especially at atomic resolution. Calculations show that the $\mathrm{X}$-ray pulse must be short enough to only probe the molecule at times before it is converted into a plasma. ${ }^{96}$ Moreover, the scattering signal must be large enough to be clearly detectable above all sources of noise, including scattered light, electric noise, and background signal.

In order to test the feasibility of single-particle diffraction of such large systems, we propose a bottom-up approach: one would perform large angle diffraction imaging on molecules containing tens of atoms in order to explore the technical challenges of such experiments. Recently, it was theoretically investigated which structural information can, in principle, be extracted from the X-ray diffraction patterns of aligned samples of small symmetric top molecules. ${ }^{97,98}$ It is clear that for such small species the signals from many molecules must be averaged. Therefore, it is important to provide samples which are dense and as clean and defined as possible to allow for experimental averaging over multiple X-ray pulses and successive image averaging. Here, the existence of the abovementined isomers turns out to be a real problem. All isomers will yield individual diffraction images that cannot be averaged over as this would obscure the structural information. Instead, the structural isomers must be spatially separated and only a

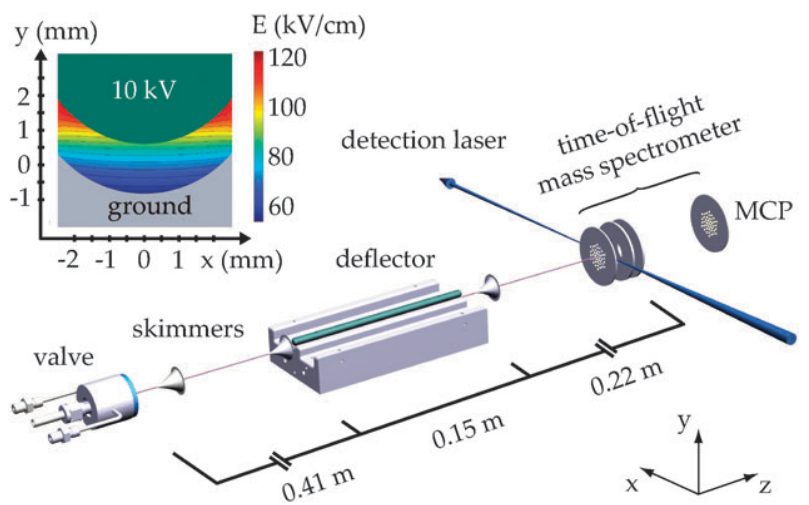

Fig. 2 Cold polar molecules in a supersonic jet are dispersed according to their effective dipole moments upon passing a strong inhomogeneous electric field. In the detection region quantum-stateand conformer-selective experiments can be conducted by changing the height of the detection laser. See text for details. 
single conformer must enter the imaging system. This isomer separation can be achieved by the different experimental approaches described in this article. Moreover, this selection will intrinsically provide samples with most population in the lowest rotational states, which can be aligned and oriented especially well using strong laser or dc electric fields (vide infra).

\section{Experimental results}

In all experiments discussed here, strong inhomogeneous electric fields are used to manipulate the motion of large neutral molecules. In the conceptually most simple setup, depicted in Fig. 2, a static electric field is used to disperse beams of polar molecules in a deflector. The experimental setup is described in detail elsewhere. ${ }^{99,100}$ In brief, a molecular beam is formed by expanding a mixture of helium (50-100 bar) and the target molecules (at a partial pressure of a few mbar) through a pulsed Even-Lavie valve ${ }^{101}$ into vacuum. During the supersonic expansion the molecules are efficiently cooled via collisions with the carrier gas to a rotational temperature of $\sim 1 \mathrm{~K}$. The cold molecular beam is collimated using two skimmers before entering a $15-\mathrm{cm}$ long electrostatic deflector. A cut through the electric field of the deflector is shown in the inset of Fig. 2. This so-called two-wire field $^{9}$ has a large gradient along the vertical $y$-axis and is homogeneous along the horizontal $x$-axis. Thus polar molecules are predominantly deflected vertically with molecules in high-field-seeking quantum states being deflected upwards. The deflected molecules then pass a third skimmer before they are intersected in the interaction region by a focused ionizing laser pulse. The height of the detection laser focus is scanned in order to measure the vertical molecular beam intensity profile. The created ions, mass-selected by a linear time-of-flight mass spectrometer, are detected using a microchannel plate detector.

The idea behind this deflection setup is straightforward: upon passing through the electrostatic deflector the species in

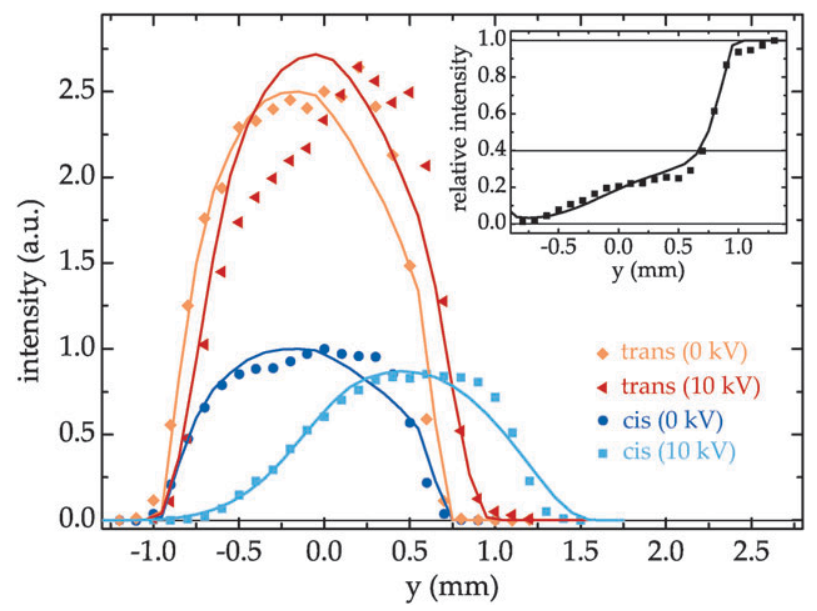

Fig. 3 Vertical molecular beam intensity profiles for cis-3AP and trans-3AP with and without high voltages applied to the deflector. Experimental data is shown as symbols, simulations are shown as solid lines. In the inset the fractional intensity of the cis conformer is shown as a function of the vertical position $y$ of the detection laser (reproduced from ref. 86). the molecular beam are dispersed according to their effective dipole moments $\mu_{\text {eff }}$. High-field-seeking molecules with a large and positive $\mu_{\text {eff }}$ end up very high in the detection region. Unpolar species, such as the carrier gas atoms or molecules in very high-lying rotational quantum states, will remain close to the molecular beam axis since they are not deflected. If molecules in low-field seeking states (i.e., states with $\mu_{\mathrm{eff}}<0$ ) are present, these molecules will reach the detection region below the molecular beam axis. We have demonstrated that this experimental setup can indeed be applied for the conformer separation of large molecules. ${ }^{86}$ Fig. 3 shows a measurement of the vertical intensity profiles for cis- and trans-3AP with and without high voltages applied to the deflector. The two conformers can be detected individually due to their distinct excitation wavelength in the one-color resonance-enhanced multiphoton ionization scheme that is used for the detection. ${ }^{102}$ Without high voltages applied to the deflector, both conformers exhibit the same spatial distribution. When $10 \mathrm{kV}$ are applied to the deflector all molecules are deflected upwards, as all quantum states of both conformers are high-field seeking at the relevant electric field strenghts (see Fig. 1). However, the deflection is stronger for the more polar cis-conformer and above $y=1 \mathrm{~mm}$ a pure sample consisting of only cis-3AP is obtained. These isolated samples of cis-3AP molecules consist exclusively of molecules in the lowest rotational quantum states, which have the largest effective dipole moments. The cis-3AP molecules in high- $J$ states have smaller effective dipole moments, comparable to those of the low- $J$ states of the trans conformer. Therefore, in the region around $y=0.75 \mathrm{~mm}$, cis-3AP molecules in high- $J$ states and trans-3AP molecules in low- $J$ states spatially overlap. Only in the lowest part of the molecular beam, below $y=-0.75 \mathrm{~mm}$ where the population of cis-3AP is completely depleted, a clean sample of the trans conformer is obtained. Note, however, that in this region the trans-3AP molecules are predominantly in high- $J$ states. These molecules are still overlaid with the $\mathrm{He}$ atoms from the carrier gas, which is not affected by the electric field. In order to isolate the trans3AP molecules in the lowest rotational states from both the cis conformer and the carrier gas, the deflection experiment can be performed with $\mathrm{Ne}$ as the carrier gas, thereby optimizing the deflection amplitudes by lowering the molecular beam velocity. Under these conditions the beam is practically devoid of cis-3AP and pure samples of the lowest, most polar states of trans-3AP can be obtained. ${ }^{103}$

One disadvantage of the electrostatic deflection technique is that it does not provide any focusing but merely disperses the molecular beam. Many applications require tightly focused laser beams in order to achieve the necessary light intensities. In these cases, a focused molecular beam would be beneficial for optimal spatial overlap between the molecular sample and the detection laser. Whereas small molecules in low-fieldseeking quantum states can be focused using static multipole fields, alternating gradient focusing is required to confine large molecules, which are high-field seeking (see section I). Electric fields suitable for AG focusing can be created most easily by placing four cylindrical high voltage electrodes symmetrically around the molecular beam axis and applying voltages as shown in Fig. 4b. In the saddle-point like electric field of 

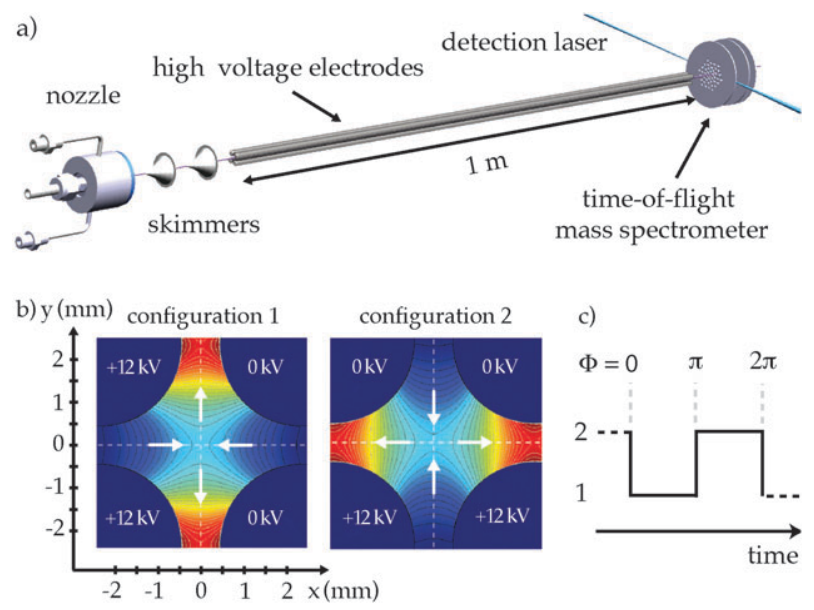

Fig. 4 (a) Scheme of the experimental setup used for alternating gradient focusing of high-field-seeking molecules. (c) Switching in a square wave pattern between the two electric field configurations shown in (b) results in a net focusing effect when the appropriate frequency is used.

configuration 1 molecules are focused towards the molecular beam axis along the $x$-direction and defocused along the $y$-direction. Switching to configuration 2, which corresponds to configuration 1 rotated by $90^{\circ}$, interchanges focusing and defocusing directions. The force acting on the molecules increases with increasing distance from the molecular beam axis. Because the molecules are on average further away from the molecular beam axis in the focusing lens compared to the defocusing lens, a net focusing effect results. The optimal switching frequency depends on the dipole moment to mass ratio. If the field is switched too slowly the molecules are pushed out of the electrodes along the defocusing direction before they are refocused by switching the high voltages. If the field is switched too rapidly the molecules see the timeaveraged potential, which is defocusing for high-field-seeking molecules. Only for a small range of frequencies AG focusing works. In other words, by choosing the appropriate switching frequency only species within a small range of dipole moment to mass ratios are transmitted. The operational principle of the device is analogous to that of the quadrupole mass filter for ions, where molecules are discriminated based on their distinct charge to mass ratios. We have demonstrated that AG focusing can also be exploited to select the individual conformers of $3 \mathrm{AP} .{ }^{104}$ At high ac frequencies, i.e., around $3 \mathrm{kHz}$, predominantly the more polar cis conformer is transmitted, whereas the less polar trans conformer is selected at lower ac frequencies $(\sim 1.5 \mathrm{kHz})$. Furthermore, similar to the deflection setup, quantum-state selectivity is achieved since the lowest quantum states for a given conformer can be focused best. In the initial experiments shown in ref. 104, the selectivity was inferior compared to the deflection experiments. However, the selectivity of the focusing setup can be considerably improved by lowering the molecular beam temperature thereby maximizing the population of the lowest rotational quantum states. Moreover, we have demonstrated the possibility to experimentally increase the resolution $\Delta \mu / \mu$ of the selector by changing the duty cycle of the square wave shown in Fig. 4(c). ${ }^{105}$ This is, effectively, the same effect as adding a dc offset in a quadrupole mass filter. ${ }^{106,107}$
In principle, a similar separation can also be achieved in the time domain. It has been shown that large molecules can be decelerated using alternating gradient decelerators. ${ }^{60} \mathrm{In}$ principle, the AG decelerator could be used for the conformer and quantum-state selection, since the deceleration process is quantum-state selective and thus intrinsically conformer selective. However, AG deceleration is technically more challenging and so far no species with multiple conformers has been decelerated.

For diffraction experiments at XFELs conformer-selected samples, prepared by one of the techniques described above, are highly desired because the presence of multiple structural isomers will prohibit analysis of the diffraction patterns. Moreover, the ideal targets for diffraction experiments are molecular samples that are also aligned or oriented in the laboratory frame. Here alignment refers to confinement of a molecule-fixed axis along a laboratory-fixed axis, and orientation refers to the molecular dipole moments pointing in a particular direction. Alignment can readily be obtained by the interaction of molecules with strong ac (laser) fields. ${ }^{108,109}$ Orientation is typically achieved through hexapole stateselection for small molecules, ${ }^{110}$ brute-force orientation, ${ }^{111,112}$ or applying mixed ac and dc electric fields. ${ }^{113-115}$ We have recently demonstrated that the quantum-state selected polar samples produced by the manipulation methods described above allow the creation of strongly aligned and oriented molecular ensembles. ${ }^{99,100}$ Similar experiments on hexapolestate-selected NO molecules in lfs states were performed using ultrashort laser pulses and moderately strong dc fields. ${ }^{116}$

To illustrate the potential of this method, we studied adiabatic alignment of 2,5-diiodobenzonitrile (DIBN) molecules, which are an interesting candidate for proof-of-principle X-ray diffraction experiments. The experimental setup for these experiments is very similar to the one shown in Fig. 2, with the only difference that now two laser pulses intersect the

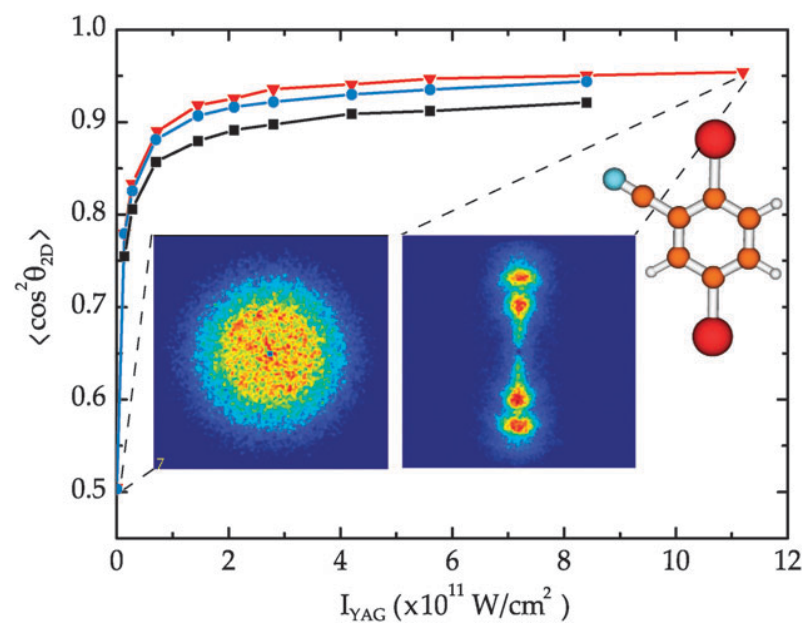

Fig. 5 Alignment of 2,5-diiodobenzonitrile as a function of the YAG laser intensity. Black squares are obtained for the undeflected molecular beam and blue circles (red triangles) correspond to a deflected sample at a height of $80 \%(50 \%)$ of the peak intensity in the deflection profile of the molecule obtained with $10 \mathrm{kV}$ applied to the deflector. Inset: $\mathrm{I}^{+}$images obtained with probe laser only (left) and with both alignment and probe laser (right). 
molecular beam in the interaction region. A $10 \mathrm{~ns}$ long YAG laser pulse is used to align the molecules and a $30 \mathrm{fs}$ long $\mathrm{Ti}$ : Sa laser pulse is employed to Coulomb explode the molecules at the peak of the YAG laser pulse. Furthermore, a velocity map imaging spectrometer is used for detection. The basic experimental observables, shown in Fig. 5, are 2D images of $\mathrm{I}^{+}$ions recorded when the molecules are irradiated with both the linearly polarized YAG pulse and the fs probe pulse. The angular distribution of the $\mathrm{I}^{+}$ions provides direct information about the spatial orientation of the C-I bond axis of the DIBN molecules. When no YAG laser pulse is employed the ion distribution is circular symmetric (see left inset of Fig. 5) and $\left\langle\cos ^{2} \theta_{2 D}\right\rangle=0.50$, where $\theta_{2 D}$ is the angle between the projection of the $\mathrm{I}^{+}$recoil velocity on the detector plane and the YAG polarization. The image changes dramatically when the YAG laser is employed. Now all $\mathrm{I}^{+}$are ejected in a narrow cone along the laser polarization axis and $\left\langle\cos ^{2} \theta_{2 D}\right\rangle=0.95$ is observed for the highest laser intensity and the most deflected molecules. From the measurements at different positions within the vertical molecular beam intensity profile it is indeed clear that the degree of alignment systematically increases with the deflection amplitude in consistency with previous studies. ${ }^{99,100}$

\section{Diffractive imaging of controlled molecular ensembles}

The conformer-selected and oriented molecular ensembles provided by state-of-the-art molecular beams and the manipulation methods described above are ideal targets for diffractive imaging experiments using novel femtosecond XFELs ${ }^{91,92}$ or ultrashort electron packets..$^{88,117,118}$ If one would take photographs of such ensembles, all molecules would look identical and they would also all be in the identical pose. Such samples allow to directly measure molecular properties - which manifest themselves in the molecule-fixed frame - in the laboratory space, i.e., the frame of measurement: the link between the two frames of reference is given by the control over the motion of the molecules demonstrated here. Possible applications of this involve the measurement of photoelectron angular distribution functions of large molecules, ${ }^{119}$ and the search for associated interferences in "diffraction from within" measurement ${ }^{120}$ (sometimes also called "photoelectron holography"121-123), which would yield detailed information on the molecular structure. Other fields which could benefit from the controlled samples include short-pulse dynamics, including impulsive alignment and the observation of the full quantum structure of rotational dynamics, high-harmonic-generation and attosecond experiments, stereochemically controlled photodissociation, half-collisons of weakly bound complexes, or generally reaction dynamics.

Here, we discuss the prospects of these samples for experiments in which we more directly take actual "photographs" of the molecules, i.e., we describe the possibility to apply these samples in diffractive imaging experiments. We propose to acquire time-resolved images of aligned and oriented individual structural isomers of molecules by coherent diffractive imaging (CDI) with XFEL pulses. The exact investigations detailed below will likely not provide new insight into the structure and dynamics of the relatively simple

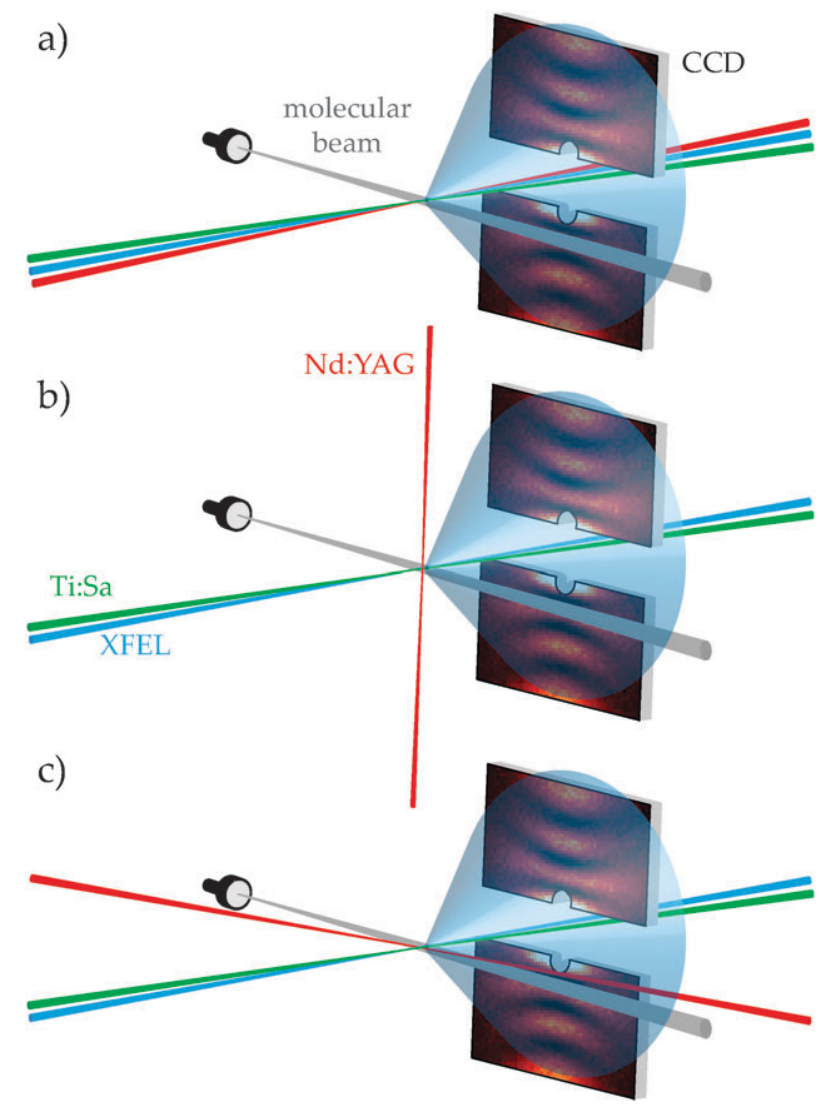

Fig. 6 Sketch of three possible experimental setups for ultrafast diffractive imaging of aligned and oriented molecular ensembles. (a) The alignment laser (red), the pump laser (green), and the X-ray (probe) laser (blue) are all co-propagating, crossing the molecular beam at right angle, and continuing through an opening between the two panels of the CCD detectors. (b) The pump laser and the X-ray laser are co-propagating, crossing the molecular beam at right angle, and continuing through an opening in the CCD detector. However, in this setup the alignment laser crosses the molecular beam and the pump and probe lasers in the interaction region also at right angles. (c) The pump laser and the X-ray laser are co-propagating, crossing the molecular beam at nearly right angle $\left(75^{\circ}\right)$, and continuing through an opening in the CCD detector. However, in this setup the alignment laser is oriented in the plane of the other beams and crosses the molecular beam at a small angle $\left(30^{\circ}\right)$ and the pump and probe lasers in the interaction region at nearly right angles $\left(-75^{\circ}\right)$.

example molecules. However, they will benchmark the proposed experimental techniques and evaluate the possibilities offered by the new instrumentation that is becoming operational right now. This includes, for example, the structural biology experiments described in section IC.

A sketch of the experimental setup is shown in Fig. 6. In all experimental approaches one would use an electric deflector to spatially separate the (polar) molecules from the atomic seed gas in order to mitigate the background that would result from scattering of X-rays. It should be realized that the background scattering of helium from a seeded molecular beam is comparable to the scattering of the seed molecules: while the scattering cross section for helium is much smaller than for the molecules, there is a strong excess in particle numbers on the order of $10^{4}$ helium atoms per molecule. 
Generally, one would record the wide-angle forwardscattered diffraction pattern, from synchronized pulsed packets of molecules in a molecular beam, on a CCD detector. Additionally, the interaction region would be intersected by an alignment laser pulse and by an ultrashort laser pulse which are also both synchronized to the XFEL pulses. Strong 1D and 3D alignment or orientation of the molecular samples in space will be induced by the alignment laser pulses and dc electric fields. ${ }^{99,109,113,124}$ Comparing the different setups sketched in Fig. 6 scheme (a) is the conceptionally simplest setup, but requires the merging of three laser beams, i.e., a pulsed nanosecond near-infrared (NIR) laser for adiabatic alignment, a NIR, visible (VIS), or ultraviolet (UV) ultrashort laser pulse inducing molecular dynamics, and the X-ray laser. This can be achieved using a holey mirror, where the X-rays are transmitted through the hole and the optical wavelengths are reflected by appropriate dielectric coatings on the mirror substrate. However, in this setup a change of the polarization axis of the alignment laser, which rotates the molecular sample in space, is identical to a rotation of the camera about the laser axis. Therefore, this setup does not allow tomographic reconstruction experiments. This possibility can be obtained by changing the alignment laser axis so that it does not coincide with the camera normal, as depicted in Fig. 6b and c. In both setups one would create a line-focus of the alignment laser (micrometers in the focused dimension and millimeters in the unfocused dimension) in order to align the full molecular ensemble probed by the $\mathrm{X}$-ray laser, i.e., the intersection column between the X-ray beam (with $\mu \mathrm{m}$ diameter) and the molecular beam (with $\mathrm{mm}$ diameter). Fig. $6 \mathrm{~b}$ would be the optimal setup regarding tomography, but would strongly interfere with secondary detectors and diagnostics, i.e., ion and electron spectrometers. These would, however, be necessary to determine and optimize spatial and temporal overlap of all pulses and to determine and measure the degree of alignment of the molecular ensemble. Moreover, it would be crucial to correlate the obtained diffraction images to molecular processes, for example, to the radiation damage characterized by the observed charge states.

We point out that we are discussing adiabatic alignment, where the X-ray scattering occurs while the molecule is in the strong ac field of the alignment laser. Laser-field-free aligned samples can be obtained using femtosecond laser methods but typically the degree of alignment is considerably lower compared to that obtained with adiabatic alignment. ${ }^{125-127}$ Also, field-free alignment occurs only in a narrow time window (few hundred femtoseconds) which may be too short to follow a reaction through its entire duration. Moreover, while the laser field could influence electronically excited states, the ground state structures of molecules, discussed here, are not significantly influenced by the off-resonant laser field. Therefore, there is no influence on the X-ray diffraction signal in the discussed limit where a molecule interacts, on average, with less than one X-ray photon. This can be different for considerably more intense X-ray pulses or for processes based on the absorption of UV/VIS/NIR or X-ray-photons, where the created excited states could be influenced by the alignment laser field. This includes, for example, studies of dynamics of electronically excited states or X-ray-ionization photoelectron measurements. The influence of these effects on the proposed dynamics studies need to be investigated, making the proposed benchmark experiments on well-known molecules even more important.

The diffraction pattern of a single molecule is the intersection of the molecular transform, i.e., the continuous 3D Fourier transform of the molecules' electron density, with the Ewald sphere. The diffraction pattern of an ensemble of gas-phase molecules is the incoherent sum of all the individual patterns, even though with an XFEL the ensemble might be illuminated with a spatially coherent beam. Thus the signal depends linearly on the number of molecules. The coherence of the X-ray pulses will lead to speckles that are of size inversely proportional to the largest intermolecular separations, but these will be much smaller than a single detector pixel and will be averaged out. Additionally, the molecules are randomly positioned-so diffracted amplitudes tend not to sum in phase - and the distribution changes shot to shot. For perfectly oriented ensembles of molecules, the accumulated diffraction pattern would, therefore, be the incoherent sum of the identical single-molecule patterns. Using the phaseretrieval algorithms of coherent diffractive imaging one can reconstruct 2D images of those patterns, in a specific view. By varying the alignment laser polarization (in setups Fig. 6b and c), and hence the angle of the alignment and orientation axis of the molecules with direction of the X-ray probe pulses, one builds up the 3D molecular transform, which can be phased to give the molecules' 3D image. Using a wavelength of $155 \mathrm{pm}$ $(8 \mathrm{keV})$ one can achieve an imaging resolution on the order of the distances between neighboring atoms in a molecule.

In initial experiments one would investigate the wide-angle diffraction imaging of simple organic molecules containing

two iodine atoms, for example, the above mentioned 2,5-diiodobenzonitrile. Its diffraction pattern will mainly consist of "double slit" like structures due to the two electron-rich iodine centers. For DIBN the I-I separation of $700 \mathrm{pm}$ is on the order of the currently available shortest wavelength of $620 \mathrm{pm}$ at LCLS, but ultrashort X-ray pulses at shorter wavelength down to $100 \mathrm{pm}$ will be available soon. In any case, clear fringes will be visible in the diffraction images. In Fig. 7 simulated diffraction images using 155 pm X-ray pulses of static samples of DIBN are shown for various degrees of 1D alignment. The simulations include photon counting statistics and no instrumental noise. In these simulations we have assumed a molecular density of $10^{10} \mathrm{~cm}^{-3}$ and $10^{13} \mathrm{X}$-ray photons/pulse focused to $10 \mu \mathrm{m}$ ( $95 \%$ intensity diameter), resulting in $\sim 160$ molecules in the $5 \mathrm{~mm}$ long interaction column of the X-ray beam and the molecular beam. Averaging over $10^{5}$ pulses, corresponding to an acquisition time of $14 \mathrm{~min}$ at a repetition rate of $120 \mathrm{~Hz}$ as available at LCLS, results in the given simulations.

In Fig. 7 simulated diffraction patterns for 2,5diiodobenzonitrile are shown: In Fig. 7(d) for a hypothetical (infeasible) perfectly 1D aligned and oriented ensemble $\left(\left\langle\cos ^{2} \theta\right\rangle=1\right)$ and different aligned samples with classical turning points for the alignment cone of (c) $\theta_{\mathrm{cl}}=10\left(\left\langle\cos ^{2} \theta\right\rangle=0.97\right)$, (b) $\theta_{\mathrm{cl}}=20\left(\left\langle\cos ^{2} \theta\right\rangle=0.88\right)$, and (a) an isotropic ensemble are shown. From these images it is obvious that the contrast in such diffraction experiments will tremendously benefit from 

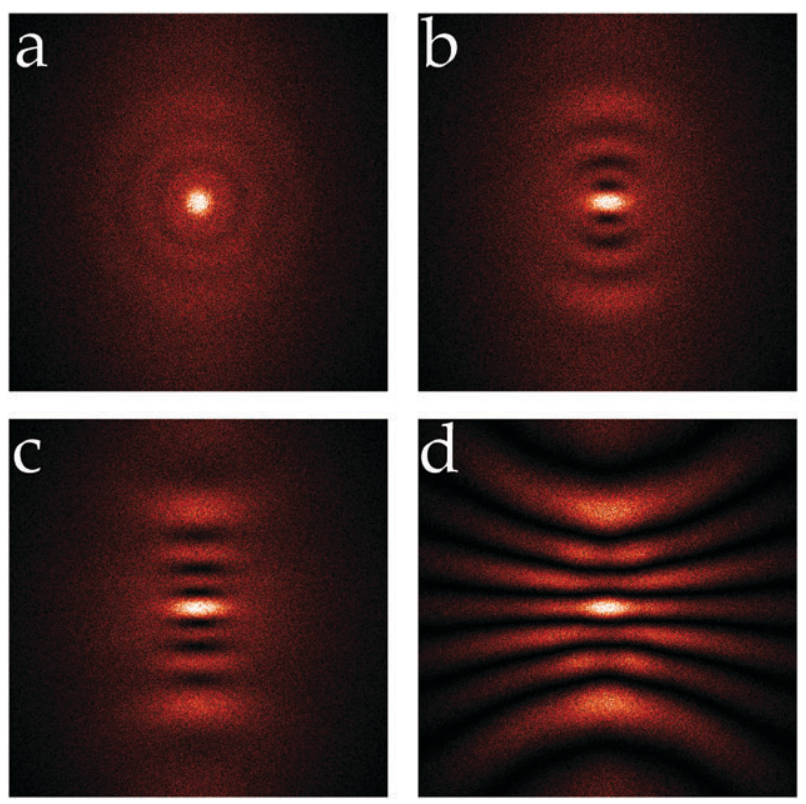

Fig. 7 Diffractive imaging patterns for 1D aligned ensembles of 2,5diiodobenzonitrile at a X-ray energies of $8 \mathrm{keV}(155 \mathrm{pm})$. (a) diffraction pattern of unaligned (isotropic) samples of DIBN (b)-(d) diffraction patterns of $1 \mathrm{D}$ aligned and oriented samples of DIBN where degrees of alignment are $\theta_{\mathrm{cl}}=20^{\circ}\left(\left\langle\cos ^{2} \theta\right\rangle=0.88\right), \theta_{\mathrm{cl}}=10^{\circ}\left(\left\langle\cos ^{2} \theta\right\rangle=0.97\right)$, and $\theta_{\mathrm{cl}}=0^{\circ}$ ("perfect" alignment), for images b, c, and d, respectively, and the orientation is always according to an up:down ratio of $10: 1$. The scattering angle at the mid-point of the CCD detector edge is $2 \theta=60^{\circ}$.
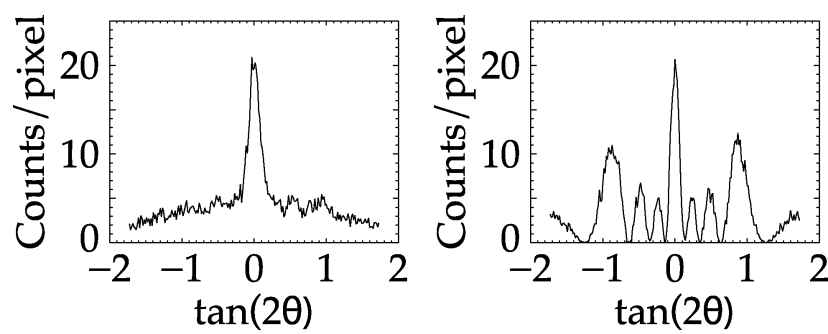

Fig. 8 Vertical lineouts of the simulated diffraction pattern of (a) unaligned and (b) 1D-aligned DIBN obtained from the images in Fig. 7 (a, d), averaged over a five pixel wide column.

strongly aligned samples, i.e., it would be extremely helpful to achieve molecular alignment of $\left\langle\cos ^{2} \theta\right\rangle>0.9$ in order to obtain maximum fringe contrast for the central vertical lineouts of these images as shown in Fig. 8. From the spacing (in reciprocal space) of two minima or maxima in the lineouts one can directly derive the dimensions of the molecule, i.e., the I-I distance in real space, by inversion of Bragg's condition. For such a simple molecule, the determined heavy-atom distance can be compared to quantum-chemical calculations and spectroscopic data, providing a detailed benchmark on the feasibility of precise structure determination of larger molecules using XFEL radiation.

In subsequent experiments one would investigate ultrafast dynamics, such as vibrational, torsional, ${ }^{128}$ or dissociation dynamics of the molecules studied. Here, we focus on dissociation dynamics, resulting in the largest structural changes. We assume that we multiply-ionize DIBN using a

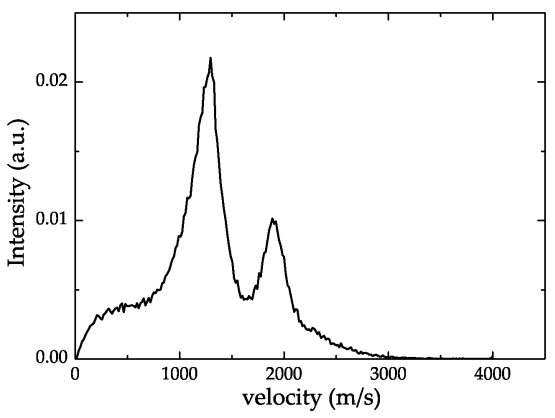

Fig. 9 Iodine ion velocity distributions obtained from Coulomb explosion imaging of diiodobenzene. ${ }^{129}$
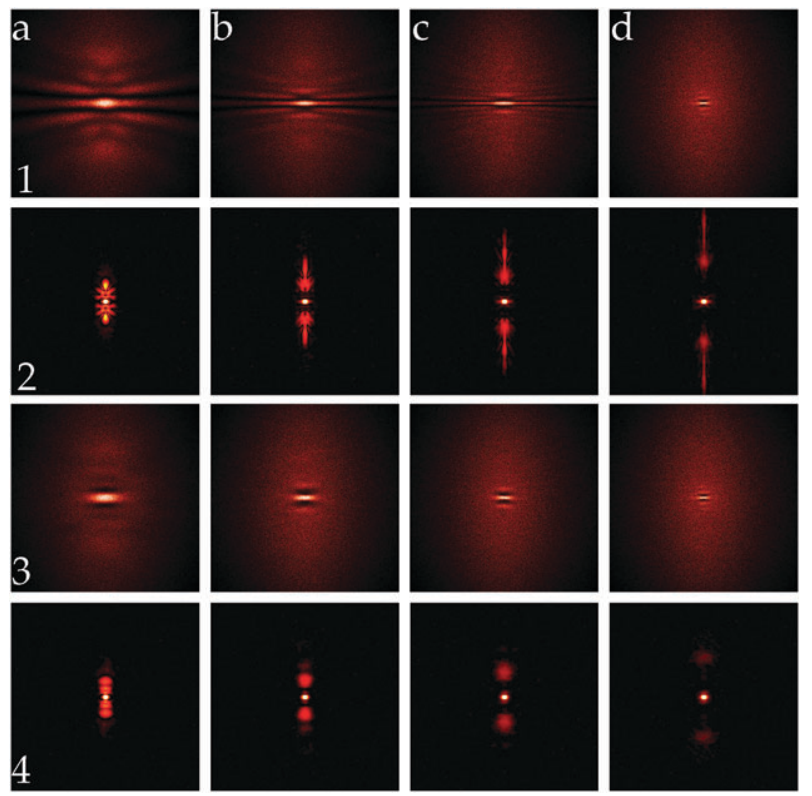

Fig. 10 Diffractive imaging patterns and their Fourier transforms for dissociating $1 \mathrm{D}$ aligned ensembles of 2,5-diiodobenzonitrile at an X-ray energy of $8 \mathrm{keV}$ (155 pm) for pump-probe delays of (a) $0 \mathrm{fs}$, (b) $250 \mathrm{fs}$, (c) $500 \mathrm{fs}$, and (d) 1 ps. Row 1 shows the diffraction patterns of perfectly aligned samples, and row 2 the corresponding Fourier transforms. Row 3 and 4 show the same images for alignment to a cone of $\pm 10^{\circ}$.

few-fs off-resonant NIR pulse, which results in Coulomb explosion and axial recoil of the two $\mathrm{I}^{+}$fragments with the velocity distribution shown in Fig. 9. Using this distribution we can simulate the diffractive imaging patterns for various timedelays between the dissociation pump pulse and the X-ray probe pulse using the same experimental parameters as above. The resulting images for originally $1 \mathrm{D}$ aligned molecular ensembles of DIBN assuming $\left\langle\cos ^{2} \theta\right\rangle=1$ and $\left\langle\cos ^{2} \theta\right\rangle=$ $0.97\left(\theta_{\mathrm{cl}}=10^{\circ}\right)$ are given in Fig. 10 rows 1 and 3 , respectively. Again, the spacings of the intensity minima and maxima in the diffraction patterns directly translate into the I-I distance. The structures of images a 1 and a 3 are somewhat washed out due to averaging over the timing jitter of the pump laser. Moreover, rows 2 and 4 of Fig. 10 show the corresponding Fourier transforms of these diffraction patterns. For clarity the square roots of the amplitudes are shown and the colorscale extends from the minimum value to one-half of the the maximum value. The separations of the heavy iodine atoms 
are directly visible and the used velocity distribution from Fig. 9 are clearly recognized. The images of the perfectly aligned samples show rich structure which is washed out for the realistic case obtained for $\left\langle\cos ^{2} \theta\right\rangle=0.97$ and, especially, for longer pump-probe delays. Nevertheless, even for the longest delay of $1 \mathrm{ps}$ and the realistic degree of alignment one can clearly define the separation of the major velocity component of the two main scattering centers. Determining these distances as a function of pump-probe delay one can, therefore, directly follow the molecular motion in real time.

In general, such experiments would fully utilize the properties of XFEL sources, i.e., the high peak brightness and short wavelengths. ${ }^{91,92}$ The X-ray pulse needs to be spatially coherent over the size of an individual molecule, but not over the size of the sample, as the intensities of individual diffraction images are summed incoherently. The available ultrashort pulses allow to obtain scattering data without blurring due to residual rotational or induced vibrational/ dissociative molecular motions, and the high pulse fluences allow to obtain scattering signals from an ensemble of gasphase molecules above experimental noise levels within relatively short times. The short wavelengths of these XFELs are required to resolve the atoms in molecular compounds. There have been many arguments and simulations on the concept of diffraction before destruction. ${ }^{96}$ It is generally understood that the intense pulses from the XFELs will lead to very strong ionization of the samples and, successively, Coulomb explosion of the molecules. However, if the X-ray pulses are short enough, all diffraction events will be over by the time the molecule considerably changes its scattering factor. The observable destruction depends on the X-ray fluence on the individual molecules and the length of the pulse. For a given photon number per pulse the destruction will increase with decreasing focus size. ${ }^{132}$ Since the molecular beams are much larger than the interaction volume and have an essentially uniform density on the scale of the X-ray focus, one can change the fluence without affecting the overall scattering intensity, as for any linear process: While the fluence, and therefore the scattering intensity per molecule, decreases quadratically with the focus diameter, at the same time the number of molecules in the interaction volume increases quadratically. This yields, nominally, the same diffraction intensity as long as the destruction is negligible. However, as soon as the fluence is so high that is causes destruction on the time-scale of the X-ray pulse duration, a decrease in the focus size will lead to a smearing out of the diffractive imaging patterns due to summing over nonequivalent molecular systems.

\section{Summary}

Using static inhomogeneous electric fields, complex polar molecules can be deflected and spatially dispersed according to their effective dipole moment, i.e., according to their quantum state. Using switched electric fields one can also actively focus or even decelerate packets of molecules in a small set of quantum states. Both approaches, deflection and focusing, can be used to prepare packets of individual structural isomers of such complex molecules. Moreover, because the methods intrinsically create very polar samples, these molecular ensembles can be aligned and oriented extremely well. Overall, these techniques allow to prepare packets of individual structural isomers that are all fixed in space due to large degrees of alignment and orientation. In addition, the electric deflection allows for complete separation of the molecular ensemble from the atomic seed gas, resulting in pure molecular samples.

We described how these samples can be exploited for diffractive imaging experiments, exemplified for ultrafast X-ray diffraction using XFELs. These experiments provide detailed benchmarks on the feasibility of coherent hard X-ray diffractive imaging of complex gas-phase molecules and the diffract-beforedestruct concept. The correctness of the extracted structural data can be compared to independently determined spectroscopic results and X-ray diffraction data from crystals. By changing the pulse length and the focus size of the X-ray pulses one can perform detailed studies of the radiation damage and its influence on the observed diffractive imaging patterns. Moreover, it will be possible to use these relatively simple diffraction patterns to test and calibrate correlation and inversion algorithms necessary for the extraction of structures of larger molecules from their diffractive imaging patterns. These experiments thus explore new paradigms in structure determination that are enabled by XFELs. Such studies will provide a path to the imaging of peptides and other complex molecules without the need for crystallization.

Moreover, we note that a number of complementary experiments would similarly benefit from the controlled samples described above. This includes experiments on molecular frame photoelectron angular distributions, ${ }^{119,120,130}$ including photoelectron holography, ${ }^{121-123}$ high-harmonic generation and molecular orbital tomography ${ }^{87}$ or electron diffraction. ${ }^{88,89}$

\section{Acknowledgements}

We thank our colleagues at the Fritz Haber Institute and the University of Aarhus for many helpful discussions and Lotte Holmegaard, Jonas L. Hansen, Jens H. Nielsen, and Sofie Louise Kragh for help with obtaining the data in Fig. 5. Expert technical support at the Fritz Haber Institute and within the Department of Molecular Physics is gratefully acknowledged. We acknowledge financial support from the Max Planck Advanced Study Group at CFEL.

\section{References}

1 L. Dunoyer, C. R. Hebd. Séances Acad. Sci., 1911, 152, 592-595.

2 O. Stern, Z. Phys., 1921, 7, 249-253.

3 W. Gerlach and O. Stern, Z. Phys., 1922, 9, 349-352, DOI: 10.1007/BF01326983.

4 H. Kallmann and F. Reiche, Z. Phys., 1921, 6, 352-375, DOI: 10.1007/BF01327996.

5 E. Wrede, Z. Phys., 1927, 44, 261-268, DOI: 10.1007/ BF01391193.

6 O. Stern, Z. Phys., 1926, 39, 751-763, DOI: 10.1007/BF01451746.

7 I. I. Rabi, J. R. Zacharias, S. Millman and P. Kusch, Phys. Rev., 1938, 53, 318, DOI: 10.1103/PhysRev.53.318.

8 I. I. Rabi, S. Millman, P. Kusch and J. R. Zacharias, Phys. Rev., 1939, 55, 526-535, DOI: 10.1103/PhysRev.55.526.

9 N. F. Ramsey, Molecular Beams, Oxford University Press, London, GB, 1956. 
10 R. Moro, X. Xu, S. Yin and W. A. de Heer, Science, 2003, 300, 1265-1269, DOI: 10.1126/science.1083247.

11 W. A. de Heer and V. V. Kresin, arXiv, 2009, 0901.4810.

12 M. Broyer, R. Antoine, I. Compagnon, D. Rayane and P. Dugourd, Phys. Scr., 2007, 76, C135-C139, DOI: 10.1088/0031-8949/76/4/N05.

13 H. Friedburg and W. Paul, Naturwissenschaften, 1951, 38, 159, DOI: $10.1007 / \mathrm{BF} 00589590$.

14 H. G. Bennewitz and W. Paul, Z. Phys., 1954, 139, 489, DOI: 10.1007/BF01374557.

15 H. G. Bennewitz, W. Paul and C. Schlier, Z. Phys., 1955, 141, 6, DOI: $10.1007 / \mathrm{BF} 01327279$.

16 J. P. Gordon, H. J. Zeiger and C. H. Townes, Phys. Rev., 1954, 95, 282-284, DOI: 10.1103/PhysRev.95.282.

17 J. P. Gordon, H. J. Zeiger and C. H. Townes, Phys. Rev., 1955, 99, 1264-1274, DOI: 10.1103/PhysRev.99.1264.

18 H. G. Bennewitz, K. H. Kramer, J. P. Toennies and W. Paul, Z. Phys., 1964, 177, 84, DOI: 10.1007/BF01375405.

19 P. R. Brooks and E. M. Jones, J. Chem. Phys., 1966, 45, 3449, DOI: $10.1063 / 1.1728128$.

20 R. J. Beuhler, R. B. Bernstein and K. H. Kramer, J. Am. Chem. Soc., 1966, 88, 5331, DOI: 10.1021/ja00974a059.

21 D. H. Parker and R. B. Bernstein, Annu. Rev. Phys. Chem., 1989, 40, 561-595, DOI: 10.1146/annurev.pc.40.100189.003021.

22 S. Stolte, Atomic and Molecular Beam Methods, Oxford University Press, New York, NY, USA, 1988 \& 1992, vol. 1 \& 2, ch. 25, pp. 631-652.

23 E. W. Kuipers, M. G. Tenner, A. Kleyn and S. Stolte, Nature, 1988, 334, 420-422, DOI: $10.1038 / 334420 \mathrm{a} 0$.

24 T. P. Rakitzis, A. J. van den Brom and M. H. M. Janssen, Science, 2004, 303, 1852-1854, DOI: 10.1126/science.1094186.

25 H. L. Bethlem, G. Berden and G. Meijer, Phys. Rev. Lett., 1999, 83, 1558-1561, DOI: 10.1103/PhysRevLett.83.1558.

26 F. M. H. Crompvoets, H. L. Bethlem, R. T. Jongma and G. Meijer, Nature, 2001, 411, 174, DOI: 10.1038/35075537.

27 H. L. Bethlem, G. Berden, F. M. H. Crompvoets, R. T. Jongma, A. J. A. van Roij and G. Meijer, Nature, 2000, 406, 491-494, DOI: $10.1038 / 35020030$

28 J. van Veldhoven, H. L. Bethlem and G. Meijer, Phys. Rev. Lett., 2005, 94, 083001, DOI: 10.1103/PhysRevLett.94.083001.

29 S. A. Meek, H. Conrad and G. Meijer, Science, 2009, 324, 1699-1702, DOI: $10.1126 /$ science. 1175975.

30 S. Y. T. van de Meerakker, H. L. Bethlem and G. Meijer, Nat. Phys., 2008, 4, 595, DOI: 10.1038/nphys1031.

31 M. T. Bell and T. P. Softley, Mol. Phys., 2009, 107, 99-132, DOI: $10.1080 / 00268970902724955$.

32 S. Y. T. van de Meerakker, H. L. Bethlem and G. Meijer, Cold Molecules: Theory, Experiment, Applications, CRC Press, Taylor\&Francis Group, 2009, ch. 14.

33 W. Ketterle and D. E. Pritchard, Appl. Phys. B: Photophys. Laser Chem., 1992, 54, 403-406, DOI: 10.1007/BF00325386.

34 W. H. Wing, Prog. Quantum Electron., 1984, 8, 181-199, DOI: 10.1016/0079-6727(84)90012-0.

35 E. D. Courant and H. S. Snyder, Ann. Phys., 1958, 3, 1-48, DOI: 10.1016/0003-4916(58)90012-5.

36 E. D. Courant, M. S. Livingston and H. S. Snyder, Phys. Rev., 1952, 88, 1190, DOI: 10.1103/PhysRev.88.1190.

37 W. Paul, Rev. Mod. Phys., 1990, 62, 531, DOI: 10.1103/ RevModPhys.62.531.

38 W. Paul and H. Steinwedel, Z. Naturforsch., Teil A, 1953, 8, 448-450.

39 W. Paul, O. Osberghaus and E. Fischer, Ein Ionenkäfig, Wirtschafts- und Verkehrministerium Nordrhein Westfalen Forschungsbericht, Westdeutscher Verlag, Köln, Germany, 1958.

40 D. Auerbach, E. E. A. Bromberg and L. Wharton, J. Chem. Phys., 1966, 45, 2160, DOI: $10.1063 / 1.1727902$.

41 D. Kakati and D. C. Lainé, Phys. Lett. A, 1967, 24, 676, DOI: 10.1016/0375-9601(67)91022-5.

42 D. Kakati and D. C. Lainé, Phys. Lett. A, 1969, 28, 786, DOI: 10.1016/0375-9601(69)90623-9.

43 D. Kakati and D. C. Lainé, J. Phys. E: Sci. Instrum., 1971, 4, 269, DOI: $10.1088 / 0022-3735 / 4 / 4 / 002$.

44 F. Günther and K. Schügerl, Z. Phys. Chem., 1972, 80, 155.

45 A. Lübbert, F. Günther and K. Schügerl, Chem. Phys. Lett., 1975, 35, 210, DOI: 10.1016/0009-2614(75)85315-2.

46 A. Lübbert, G. Rotzoll and F. Günther, J. Chem. Phys., 1978, 69, 5174, DOI: $10.1063 / 1.436464$.
47 T. Junglen, T. Rieger, S. A. Rangwala, P. W. H. Pinkse and G. Rempe, Phys. Rev. Lett., 2004, 92, 223001, DOI: 10.1103/ PhysRevLett.92.223001

48 T. E. Wall, S. Armitage, J. J. Hudson, B. E. Sauer, J. M. Dyne, E. A. Hinds and M. R. Tarbutt, Phys. Rev. A: At., Mol., Opt. Phys., 2009, 80, 043407, DOI: 10.1103/PhysRevA.80.043407.

49 D. H. H. Al-Amiedy and D. C. Lainé, Phys. Lett. A, 1978, 66, 94-96, DOI: 10.1016/0375-9601(78)90005-1.

50 D. C. Lainé and R. Sweeting, Phys. Lett. A, 1971, 34, 144, DOI: 10.1016/0375-9601(71)90795-X.

51 J. C. Helmer, F. B. Jacobus and P. A. Sturrock, J. Appl. Phys., 1960, 31, 458, DOI: 10.1063/1.1735608.

52 K.-R. Chien, P. B. Foreman, K. H. Castleton and S. G. Kukolich, Chem. Phys., 1975, 7, 161, DOI: 10.1016/0301-0104(75)85038-5.

53 H.-J. Loesch, Chem. Phys., 1996, 207, 427, DOI: 10.1016/03010104(95)00447-5.

54 H. J. Loesch and B. Scheel, Phys. Rev. Lett., 2000, 85, 2709, DOI: 10.1103/PhysRevLett.85.2709.

55 R. Wolfgang, Sci. Am., 1968, 219, 44.

56 E. E. A. Bromberg, PhD thesis, University of Chicago, Chicago, IL, USA, 1972.

57 H. L. Bethlem, A. J. A. van Roij, R. T. Jongma and G. Meijer, Phys. Rev. Lett., 2002, 88, 133003, DOI: 10.1103 PhysRevLett.88.133003.

58 H. L. Bethlem, M. R. Tarbutt, J. Küpper, D. Carty, K. Wohlfart, E. A. Hinds and G. Meijer, J. Phys. B: At., Mol. Opt. Phys., 2006, 39, R263-R291, DOI: 10.1088/0953-4075/39/16/R01.

59 M. R. Tarbutt, H. L. Bethlem, J. J. Hudson, V. L. Ryabov, V. A. Ryzhov, B. E. Sauer, G. Meijer and E. A. Hinds, Phys. Rev. Lett., 2004, 92, 173002, DOI: 10.1103/PhysRevLett.92.173002.

60 K. Wohlfart, F. Grätz, F. Filsinger, H. Haak, G. Meijer and J. Küpper, Phys. Rev. A: At., Mol., Opt. Phys., 2008, 77, 031404, DOI: 10.1103 /PhysRevA.77.031404.

61 K. Wohlfart, F. Filsinger, F. Grätz, J. Küpper and G. Meijer, Phys. Rev. A: At., Mol., Opt. Phys., 2008, 78, 033421, DOI: 10.1103/PhysRevA.78.033421.

62 K. Wohlfart, Dissertation, Free University, Berlin, Germany, 2008.

63 H. Stapelfeldt, H. Sakai, E. Constant and P. B. Corkum, Phys. Rev. Lett., 1997, 79, 2787-2790.

64 B. S. Zhao, H. S. Chung, K. Cho, S. H. Lee, S. Hwang, J. Yu, Y. H. Ahn, J. Y. Sohn, D. S. Kim, W. K. Kang and D. S. Chung, Phys. Rev. Lett., 2000, 85, 2705-2708, DOI: 10.1103 PhysRevLett.85.2705.

65 R. Fulton, A. I. Bishop and P. F. Barker, Phys. Rev. Lett., 2004, 93, 243004, DOI: 10.1103/PhysRevLett.93.243004.

66 H. Odashima, S. Merz, K. Enomoto, M. Schnell and G. Meijer, Phys. Rev. Lett., 2010, 104, 253001, DOI: 10.1103/PhysRevLett.104.253001.

67 Special issue "Molecular physics of building blocks of life under isolated or defined conditions", Eur. Phys. J. D, 2002, 20, 309-626.

68 Special issue "Bio-active molecules in the gas phase", Phys. Chem. Chem. Phys., 2004, 6, 2543-2890, DOI: 10.1039/b405201n.

69 M. S. de Vries and P. Hobza, Annu. Rev. Phys. Chem., 2007, 58, 585-612, DOI: 10.1146/annurev.physchem.57.032905.104722.

70 S. K. Kim, W. Lee and D. R. Herschbach, J. Phys. Chem., 1996, 100, 7933-7937, DOI: $10.1021 /$ jp960635d.

71 F. Piuzzi, M. Mons, I. Dimicoli, B. Tardivel and Q. Zhao, Chem. Phys., 2001, 270, 205-214, DOI: 10.1016/S0301-0104(01)00393-7.

72 M. N. Blom, I. Compagnon, N. C. Polfer, G. von Helden, G. Meijer, S. Suhai, B. Paizs and J. Oomens, J. Phys. Chem. A, 2007, 111, 7309-7316.

73 R. D. Suenram and F. J. Lovas, J. Am. Chem. Soc., 1980, 102, 7180-7184, DOI: $10.1021 / \mathrm{ja} 00544 \mathrm{a} 002$.

74 T. R. Rizzo, Y. D. Park, L. Peteanu and D. H. Levy, J. Chem. Phys., 1985, 83, 4819-4820, DOI: 10.1063/1.449009.

75 E. Nir, K. Kleinermanns and M. S. de Vries, Nature, 2000, 408, 949-951, DOI: $10.1038 / 35050053$.

76 L. C. Snoek, E. G. Robertson, R. T. Kroemer and J. P. Simons, Chem. Phys. Lett., 2000, 321, 49-56, DOI: 10.1016/S00092614(00)00320-1.

77 J. M. Bakker, L. M. Aleese, G. Meijer and G. von Helden, Phys. Rev. Lett., 2003, 91, 203003, DOI: 10.1103/PhysRevLett.91.203003.

78 F. Dong and R. E. Miller, Science, 2002, 298, 1227-1230, DOI: $10.1126 /$ science. 1076947.

79 A. Lesarri, E. J. Cocinero, J. C. Lopez and J. L. Alonso, Angew. Chem., Int. Ed., 2004, 43, 605-610, DOI: 10.1002/anie.200352543. 
80 J. A. Reese, T. V. Nguyen, T. M. Korter and D. W. Pratt, J. Am. Chem. Soc., 2004, 126, 11387-11392, DOI: 10.1021/ja0469683.

81 F. Filsinger, K. Wohlfart, M. Schnell, J.-U. Grabow and J. Küpper, Phys. Chem. Chem. Phys., 2008, 10, 666-673, DOI: $10.1039 / \mathrm{b} 711888 \mathrm{k}$.

82 G. von Helden, T. Wyttenbach and M. T. Bowers, Science, 1995, 267, 1483-1485, DOI: $10.1126 /$ science.267.5203.1483.

83 M. Jarrold, Phys. Chem. Chem. Phys., 2007, 9, 1659-1671, DOI: 10.1039/b612615d.

84 B. C. Dian, A. Longarte and T. S. Zwier, Science, 2002, 296, 2369-2373, DOI: 10.1126/science. 1071563.

85 U. Erlekam, M. Frankowski, G. von Helden and G. Meijer, Phys. Chem. Chem. Phys., 2007, 9, 3786-3789, DOI: 10.1039/b703571c.

86 F. Filsinger, J. Küpper, G. Meijer, J. L. Hansen, J. Maurer, J. H. Nielsen, L. Holmegaard and H. Stapelfeldt, Angew. Chem., Int. Ed., 2009, 48, 6900-6902, DOI: 10.1002/anie.200902650.

87 J. Itatani, J. Levesque, D. Zeidler, H. Niikura, H. Pépin, J. C. Kieffer, P. B. Corkum and D. M. Villeneuve, Nature, 2004, 432, 867-871, DOI: 10.1038 /nature03183.

88 J. C. Williamson, J. M. Cao, H. Ihee, H. Frey and A. H. Zewail, Nature, 1997, 386, 159-162.

89 B. J. Siwick, J. R. Dwyer, R. E. Jordan and R. J. D. Miller, Science, 2003, 302, 1382-1385.

90 H. N. Chapman, A. Barty, M. J. Bogan, S. Boutet, S. Frank, S. P. Hau-Riege, S. Marchesini, B. W. Woods, S. Bajt, W. H. Benner, L. W. A., E. Plönjes, M. Kuhlmann, R. Treusch, S. Düsterer, T. Tschentscher, J. R. Schneider, E. Spiller, T. Möller, C. Bostedt, M. Hoener, D. A. Shapiro, K. O. Hodgson, D. van der Spoel, F. Burmeister, M. Bergh, C. Caleman, G. Huldt, M. M. Seibert, F. R. N. C. Maia, R. W. Lee, A. Szöke, N. Timneanu and J. Hajdu, Nat. Phys., 2006, 2, 839-843, DOI: 10.1038/nphys461.

91 M. Altarelli, R. Brinkmann, M. Chergui, W. Decking, B. Dobson, S. Düsterer, G. Grübel, W. Graeff, H. Graafsma, J. Hajdu, J. Marangos, J. Pflüger, H. Redlin, D. Riley, I. Robinson, J. Rossbach, A. Schwarz, K. Tiedtke, T. Tschentscher, I. Vartaniants, H. Wabnitz, H. Weise, R. Wichmann, K. Witte, A. Wolf, M. Wulff and M. Yurkov, The Technical Design Report of the European XFEL, Desy technical report, 2007.

92 P. Emma, R. Akre, J. Arthur, R. Bionta, C. Bostedt, J. Bozek, A. Brachmann, P. Bucksbaum, R. Coffee, F. J. Decker, Y. Ding, D. Dowell, S. Edstrom, A. Fisher, J. Frisch, S. Gilevich, J. Hastings, G. Hays, P. Hering, Z. Huang, R. Iverson, H. Loos, M. Messerschmidt, A. Miahnahri, S. Moeller, H. D. Nuhn, G. Pile, D. Ratner, J. Rzepiela, D. Schultz, T. Smith, P. Stefan, H. Tompkins, J. Turner, J. Welch, W. White, J. Wu, G. Yocky and J. Galayda, Nat. Photonics, 2010, 4, 641-647, DOI: 10.1038/nphoton.2010.176.

93 G. Huldt, A. Szoke and J. Hajdu, J. Struct. Biol., 2003, 144, 219-227, DOI: 10.1016/j.jsb.2003.09.025.

94 Elements of Chemical Philosophy (1812), in The Collected Works of Sir Humphry Davy, ed. J. Davy, Smith, Elder and Co., London, 1839-1840, vol. 4, p. 37.

95 J. C. H. Spence and R. B. Doak, Phys. Rev. Lett., 2004, 92 198102, DOI: 10.1103/PhysRevLett.92.198102.

96 R. Neutze, R. Wouts, D. van der Spoel, E. Weckert and J. Hajdu, Nature, 2000, 406, 752-757, DOI: 10.1038/35021099.

97 P. J. Ho, D. Starodub, D. K. Saldin, V. L. Shneerson, A. Ourmazd and R. Santra, J. Chem. Phys., 2009, 131, 131101, DOI: 10.1063/ 1.3245404 .

98 S. Pabst, P. J. Ho and R. Santra, Phys. Rev. A: At., Mol., Opt Phys., 2010, 81, 043425, DOI: 10.1103/PhysRevA.81.043425.

99 L. Holmegaard, J. H. Nielsen, I. Nevo, H. Stapelfeldt, F. Filsinger, J. Küpper and G. Meijer, Phys. Rev. Lett., 2009, 102, 023001, DOI: 10.1103/PhysRevLett.102.023001.

100 F. Filsinger, J. Küpper, G. Meijer, L. Holmegaard, J. H. Nielsen, I. Nevo, J. L. Hansen and H. Stapelfeldt, J. Chem. Phys., 2009, 131, 064309, DOI: $10.1063 / 1.3194287$

101 M. Hillenkamp, S. Keinan and U. Even, J. Chem. Phys., 2003, 118, 8699-8705, DOI: 10.1063/1.1568331.

102 C. Unterberg, A. Gerlach, A. Jansen and M. Gerhards, Chem. Phys., 2004, 304, 237-244, DOI: 10.1016/j.chemphys.2004.06.036.

103 F. Filsinger, PhD thesis, Radboud Universiteit, Nijmegen, The Netherlands, 2010.
104 F. Filsinger, U. Erlekam, G. von Helden, J. Küpper and G. Meijer, Phys. Rev. Lett., 2008, 100, 133003, DOI: 10.1103/ PhysRevLett.100.133003.

105 F. Filsinger, S. Putzke, H. Haak, G. Meijer and J. Küpper, Phys. Rev. A, 2010, 82, 052513 .

106 J. A. Richards, R. M. Huey and J. Hiller, Proceedings of the Institution of Radio and Electronics Engineers, Australia, 1971, pp. 321-322.

107 J. A. Richards, R. M. Huey and J. Hiller, Int. J. Mass Spectrom. Ion Phys., 1973, 12, 317-339, DOI: 10.1016/0020-7381(73)80102-0.

108 B. Friedrich and D. Herschbach, Phys. Rev. Lett., 1995, 74, 4623-4626, DOI: 10.1103/PhysRevLett.74.4623.

109 H. Stapelfeldt and T. Seideman, Rev. Mod. Phys., 2003, 75, 543-557, DOI: 10.1103/RevModPhys.75.543.

110 J. Reuss, Atomic and Molecular Beam Methods, Oxford University Press, New York, NY, USA, 1988, vol. 1, ch. 11, pp. 276-292.

111 B. Friedrich and D. R. Herschbach, Nature, 1991, 353, 412-414, DOI: $10.1038 / 353412 \mathrm{a} 0$.

112 H. J. Loesch and A. Remscheid, J. Chem. Phys., 1990, 93, 4779, DOI: $10.1063 / 1.458668$.

113 B. Friedrich and D. Herschbach, J. Phys. Chem. A, 1999, 103, 10280-10288, DOI: 10.1021/jp992131w.

114 H. Sakai, S. Minemoto, H. Nanjo, H. Tanji and T. Suzuki, Phys. Rev. Lett., 2003, 90, 083001, DOI: 10.1103/PhysRevLett.90.083001.

115 U. Buck and M. Fárník, Int. Rev. Phys. Chem., 2006, 25, 583-612, DOI: $10.1080 / 01442350600847746$

116 O. Ghafur, A. Rouzee, A. Gijsbertsen, W. K. Siu, S. Stolte and M. J. J. Vrakking, Nat. Phys., 2009, 5, 289-293, DOI: 10.1038/ nphys 1225.

117 B. J. Siwick, J. R. Dwyer, R. E. Jordan and R. J. D. Miller, J. Appl. Phys., 2002, 92, 1643-1648, DOI: 10.1063/1.1487437.

118 P. Reckenthaeler, M. Centurion, W. Fuss, S. A. Trushin, F. Krausz and E. E. Fill, Phys. Rev. Lett., 2009, 102, 213001, DOI: 10.1103/PhysRevLett.102.213001.

119 L. Holmegaard, J. L. Hansen, L. Kalhøj, S. L. Kragh, H. Stapelfeldt, F. Filsinger, J. Küpper, G. Meijer, D. Dimitrovski, M. Abu-samha, C. P. J. Martiny and L. B. Madsen, Nat. Phys., 2010, 6, 428, DOI: 10.1038/nphys1666.

120 A. Landers, T. Weber, I. Ali, A. Cassimi, M. Hattass, O. Jagutzki, A. Nauert, T. Osipov, A. Staudte, M. Prior, H. Schmidt-Böcking, C. Cocke and R. Dörner, Phys. Rev. Lett., 2001, 87, 013002, DOI: 10.1103/PhysRevLett.87.013002.

121 A. Szöke, AIP Conf. Proc., 1986, 147, 361.

122 J. Barton, Phys. Rev. Lett., 1988, 61, 1356-1359.

123 F. Krasniqi, B. Najjari, L. Strüder, D. Rolles, A. Voitkiv and J. Ullrich, Phys. Rev. A: At., Mol., Opt. Phys., 2010, 81, 033411, DOI: $10.1103 /$ PhysRevA.81.033411.

124 I. Nevo, L. Holmegaard, J. H. Nielsen, J. L. Hansen, H. Stapelfeldt, F. Filsinger, G. Meijer and J. Küpper, Phys. Chem. Chem. Phys., 2009, 11, 9912-9918, DOI: 10.1039/b910423b.

125 P. M. Felker, J. S. Baskin and A. H. Zewail, J. Phys. Chem., 1986, 90, 724-728.

126 F. Rosca-Pruna and M. J. J. Vrakking, Phys. Rev. Lett., 2001, 87, 153902, DOI: 10.1103/PhysRevLett.87.153902.

127 K. F. Lee, D. M. Villeneuve, P. B. Corkum, A. Stolow and J. G. Underwood, Phys. Rev. Lett., 2006, 97, 173001, DOI: 10.1103/PhysRevLett.97.173001.

128 C. B. Madsen, L. B. Madsen, S. S. Viftrup, M. P. Johansson, T. B. Poulsen, L. Holmegaard, V. Kumarappan, K. Jørgensen and H. Stapelfeldt, Phys. Rev. Lett., 2009, 102, 073007, DOI: 10.1103/ PhysRevLett.102.073007.

129 V. Kumarappan, C. Z. Bisgaard, S. S. Viftrup, L. Holmegaard and H. Stapelfeldt, J. Chem. Phys., 2006, 125, 194309, DOI: $10.1063 / 1.2388273$.

130 L. Nugent-Glandorf, M. Scheer, D. Samuels, A. Mulhisen, E. Grant, X. Yang, V. Bierbaum and S. Leone, Phys. Rev. Lett., 2001, 87, 193002, DOI: 10.1103/PhysRevLett.87.193002.

131 In fact, Stern states in a footnote of his initial paper on space quantization $^{2}$ that its publication was motivated by Kallmann and Reiche's article, of which he had received the galley proofs.

132 In principle, similar investigations can be performed by attenuating the X-ray beam, which would, however, result not only in less destruction but also in similarly decreased signal intensities. 